\title{
Carteolol Hydrochloride Suppresses the Generation of Reactive Oxygen Species and Rescues Cell Death After Ultraviolet Irradiation of Cultured Lens Epithelial Cells
}

\author{
Yuichi Kaji*, Takahiro Kiuchi and Tetsuro Oshika
}

\author{
Department of Ophthalmology, University of Tsukuba Institute of Clinical Medicine, Tennoudai 1-1-1, Tsukuba, Ibaraki \\ 305-8575, Japan
}

\begin{abstract}
Introduction: Anti-oxidant activities of adrenergic $\beta$-blockers are proposed in various organs. The aim of the present study was to investigate the effect of carteolol hydrochloride, an adrenergic $\beta$-blocker, on the production of reactive oxygen species (ROS) and the viable cell number after ultraviolet irradiation of cultured lens epithelial cells (LECs).

Materials and Methodology: Cultured LECs were exposed to $0,10^{-5}, 10^{-4}$, and $10^{-3} \mathrm{M}$ carteolol hydrochloride for 30 min followed by ultraviolet B (UVB) irradiation at intensity of 100, 200, or $400 \mathrm{~mJ} / \mathrm{cm}^{2}$. The amount of ROS in the LECs was measured using dichlorodihydrofluorescein at $30 \mathrm{~min}$ after exposure to UVB. In addition, the number of living LECs was counted at $15 \mathrm{~h}$ after exposure to UVB.

Results: Exposure to $10^{-3} \mathrm{M}$ carteolol hydrochloride significantly decreased the amount of ROS after exposure to UVB at intensities of 100,200 , and $400 \mathrm{~mJ} / \mathrm{cm}^{2}$. In addition, $10^{-3} \mathrm{M}$ carteolol hydrochloride significantly increased the viable cell number after exposure to UVB at $400 \mathrm{~mJ} / \mathrm{cm}^{2}$. However, $10^{-4}$ and $10^{-5} \mathrm{M}$ carteolol hydrochloride had no significant effect on ROS or the viable cell number in LECs.
\end{abstract}

Discussions: Carteolol hydrochloride protects LECs against UVB irradiation by inhibiting the intracellular production of ROS.

Keywords: Reactive oxygen species, carteolol hydrochloride, ultraviolet, antioxidant, lens epithelial cells.

\section{INTRODUCTION}

Various population-based studies have clearly shown that the prevalence of glaucoma increases with age $[1,2]$. This means that considerable numbers of patients with glaucoma also have cataracts or have already received cataract surgery. In addition, long-term topical application of anti-glaucoma drugs is needed in most of the patients with glaucoma. At present, however, little is known about the effect of antiglaucoma drugs on cataract formation. Accordingly, the effect of these drugs on the development of cataracts should be investigated.

$\beta$-Adrenergic blockers are widely used to treat glaucoma and ocular hypertension as well as hypertension and cardiac arrhythmia. Various papers have shown that $\beta$-adrenergic blockers serve as an antioxidant in experimental models. For example, antioxidant effects are reported for timolol, betaxolol, and niparadiol on cultured retinal ganglion cells [3]; for metipranolol and desacetyl metipranolol on the brain [4]; and for arotinolol on muscles [5]. However, the minute molecular mechanisms of antioxidant activities of $\beta$ adrenergic blockers are still unclear. Among the various $\beta$ adrenergic blockers, molecular mechanism of antioxidant activity of carteolol hydrochloride (5-(3-tert-butylamino-2hydroxy)propoxy-3,4-dihydro-2(1H)-quinolinone monohydro-

*Address correspondence to this author at the Department of Ophthalmology, University of Tsukuba Institute of Clinical Medicine, Tennoudai 1-1-1, Tsukuba, Ibaraki 305-8575, Japan; Tel: +81-29-853-3148; Fax: +81-29-8533148; E-mail: kajiyuichi@gmail.com chloride) is partially revealed $[6,7]$. Carteolol hydrochloride is one of the $\beta$-adrenergic blockers and widely used to treat cardiac arrhythmia, glaucoma, and ocular hypertension $[8$, 9]. The reported antioxidant properties of carteolol hydrochloride have, however, differed among the previous studies. Tanito et al. have reported that carteolol hydrochloride has antioxidant properties in the cornea and retina $[6,10]$, but not in retinal ganglion cells, the brain, or muscles $[3,5]$. This indicates that the antioxidant properties of this chemical depend on the type of cell and the manner in which oxidative stress is induced. For this reason, the antioxidant activities of carteolol hydrochloride should be examined in various ocular tissues.

In the present study, we attempted to reveal the effect of carteolol hydrochloride on cataract formation. However, it is practically difficult to evaluate the effect of the topical application of carteolol hydrochloride on cataract formation over the long term (years) using in vivo models. Recently, ultraviolet irradiation of cultured lens epithelial cells (LECs) has been used as an in vitro model of cataract formation [11]. Ultraviolet light is one of the important accelerators of cataract formation $[12,13]$. In addition, ultraviolet irradiation of cultured LECs in vitro is known to induce various changes, such as racemization and glycation of crystalline molecules, apoptosis, activation of p53[14], and induction of reactive oxygen species (ROS) [15], that are closely related to the development of cataracts in vivo. For these reasons, we examined the effect of carteolol hydrochloride on the number of living cells and intracellular 
ROS after ultraviolet irradiation of cultured lens epithelial cells.

\section{MATERIALS AND METHODOLOGY}

\section{Cells}

Human lens epithelial cells (LECs), line SRA01/04, were purchased from the RIKEN Cell Bank (Tsukuba, Ibaraki, Japan). The LECs were cultured in $60-\mathrm{mm}$ culture dishes (Falcon, Lincoln Park, NJ) in Dulbecco's modified Eagle's medium (DMEM) supplemented with 15\% fetal bovine serum (FBS), L-glutamine (2 $\mathrm{mM})$, penicillin (100 units $/ \mathrm{mL})$, and streptomycin $(0.1 \mathrm{mg} / \mathrm{mL})$ in a humidified atmosphere containing $5 \% \mathrm{CO}_{2}$ at $37^{\circ} \mathrm{C}$. After the LECs had reached confluence, a portion was subcultured in a 96-well culture plate (Falcon).

\section{Chemicals}

Carteolol hydrochloride was obtained from the Tokushima Research Institute, Otsuka Pharmaceutical Co., Ltd. 2',7'-Dichlorodihydrofluorescein diacetate were purchased from Sigma.

\section{Reactive Oxygen Species After Exposure to UVB}

The LECs were seeded at 10,000 cells/well in96-well plates. Twelve hours after seeding, non-adherent LECs were washed away with the cultured medium. Carteolol hydrochloride was added to the culture medium at final concentrations of $0,10^{-5}, 10^{-4}$, and $10^{-3} \mathrm{M}$ and left for 30 min. Each well was then washed three times with phosphatebuffered saline ( $\mathrm{pH}$ 7.4) (PBS) followed by the addition of $100 \mu \mathrm{l}$ of culture medium without carteolol hydrochloride. The cells were exposed to ultraviolet B (UVB) $(0,100,200$, or $400 \mathrm{~mJ} / \mathrm{cm}^{2}$ at an exposure time of 45 seconds per 100 $\mathrm{mJ} / \mathrm{cm}^{2}$ ) using a UV transilluminator (Model UVM-57, with a peak at $302 \mathrm{~nm}$; UVP, Inc., Upland, CA). The intensity of UVB was measured using a UV radiometer (SUV-T; Toray Techno Co., Japan) at the bottom of the culture plate. The UV-irradiated LECs were treated with $10 \mu \mathrm{M}$ of $2^{\prime}, 7^{\prime}-$ dichlorodihydrofluorescein diacetate in PBS for $30 \mathrm{~min}$. Thereafter, the medium was discarded and the cells were washed with PBS. The fluorescence intensity was determined using a Varioskan microplate reader (Thermo Electric Corporation, Vantaa, Finland) at excitation and emission wavelengths of $485 \mathrm{~nm}$ and $535 \mathrm{~nm}$, respectively. UVB-induced intracellular ROS were also confirmed using a fluorescence microscope (ECLIPSE 80i; Nikon, Tokyo, Japan) equipped with a cooled CCD camera (DXM1200C; Nikon).

\section{Cell Viability Measurement After Exposure to UVB}

Pretreatment with carteolol hydrochloride and UVB irradiation was performed as described above. Fifteen hours after the UVB irradiation, non-viable cells were removed by washing three times with PBS. Then the attached cells were removed by exposing each well to $0.25 \mathrm{~mL}$ of trypsin-EDTA (GIBCO, Grand Island, NY) for 20 minutes at $37^{\circ} \mathrm{C}$. Isoton solution (American Scientific Products, McGraw, IL) was added to the cell suspension to a final volume of $10 \mathrm{~mL}$, and the cell number of each well was counted with a Coulter counter (type Z1). The experiments were repeated 8 times in each condition.

\section{Statistical Analysis}

The intensity of fluorescence and the cell viability are expressed as the mean \pm SD. The sample size for each group was 16 , and we repeated each experiment. The data for all test samples were analyzed using a one-way ANOVA and compared using the Dunnett test. A $P$ value of $<0.05$ was considered statistically significant.

\section{RESULTS}

\section{Visualization of the Amounts of Reactive Oxygen Species in Lens Epithelial Cells}

Fig. (1) shows an example of the fluorescence in LECs treated with $2^{\prime}, 7^{\prime}$-dichlorodihydrofluorescein diacetate after $200 \mathrm{~mJ} / \mathrm{cm}^{2}$ of UVB irradiation. Without exposure to UVB, the LECs exhibited almost no fluorescence. In contrast, intense fluorescence was observed in the cytoplasm and nuclei of the LECs after exposure to UVB. The intensity of fluorescence was reduced in the LECs pretreated with $10^{-3}$ $\mathrm{M}$ carteolol hydrochloride. All the images were taken with the same exposure time $(20 \mathrm{msec})$ and visualized identically.

\section{Quantification of Reactive Oxygen Species in Lens Epithelial Cells After Exposure to Ultraviolet Light}

Fig. (2A) shows the intensity of fluorescence in the LECs treated with $2^{\prime}, 7^{\prime}$-dichlorodihydrofluorescein diacetate after exposure to UVB. The intensity of fluorescence was increased by the UVB irradiation in a dose-dependent manner. Fig. (2B-D) show the effect of carteolol hydrochloride on the intensity of fluorescence after exposure to 100,200 , and $400 \mathrm{~mJ} / \mathrm{cm}^{2}$ of UVB, respectively. The intensity of fluorescence decreased with an increase in carteolol hydrochloride concentration. In addition, the intensity of fluorescence decreased significantly $(\mathrm{P}<0.01)$ in cells treated with $10^{-3} \mathrm{M}$ carteolol hydrochloride.

\section{Viability of Lens Epithelial Cells After Exposure to Ultraviolet Light}

Fig. (3A) shows the number of viable cells after exposure to UVB. Irradiation with UVB decreased the number of viable LECs significantly in a dose-dependent manner. Fig. (3B-D) show the effect of carteolol hydrochloride on the number of viable cells after exposure to 100,200 , and 400 $\mathrm{mJ} / \mathrm{cm}^{2}$ of UVB, respectively. No significant effect was observed on the number of viable cells after exposure to 100 $\mathrm{mJ} / \mathrm{cm}^{2}$ of UVB irradiation (Fig. 3B). In contrast, the number of viable LECs increased significantly when these cells were pretreated with $10^{-3} \mathrm{M}$ carteolol hydrochloride and received 200 and $400 \mathrm{~mJ} / \mathrm{cm}^{2}$ of UVB irradiation (Fig. 3C, D). Pretreatment with carteolol hydrochloride at concentrations of $10^{-4}$ and $10^{-5}$ had no significant effect on the viability of LECs that had received 200 or $400 \mathrm{~mJ} / \mathrm{cm}^{2}$ of UVB irradiation.

\section{DISCUSSION}

The purpose of the present study was to investigate the antioxidant properties of carteolol hydrochloride on LECs after irradiation with ultraviolet light. The results of this study showed that carteolol hydrochloride $\left(10^{-3} \mathrm{M}\right)$ had protective effects on the LECs (increased the number of viable cells) by inhibiting the production of ROS. These results indicate that carteolol hydrochloride may have a 

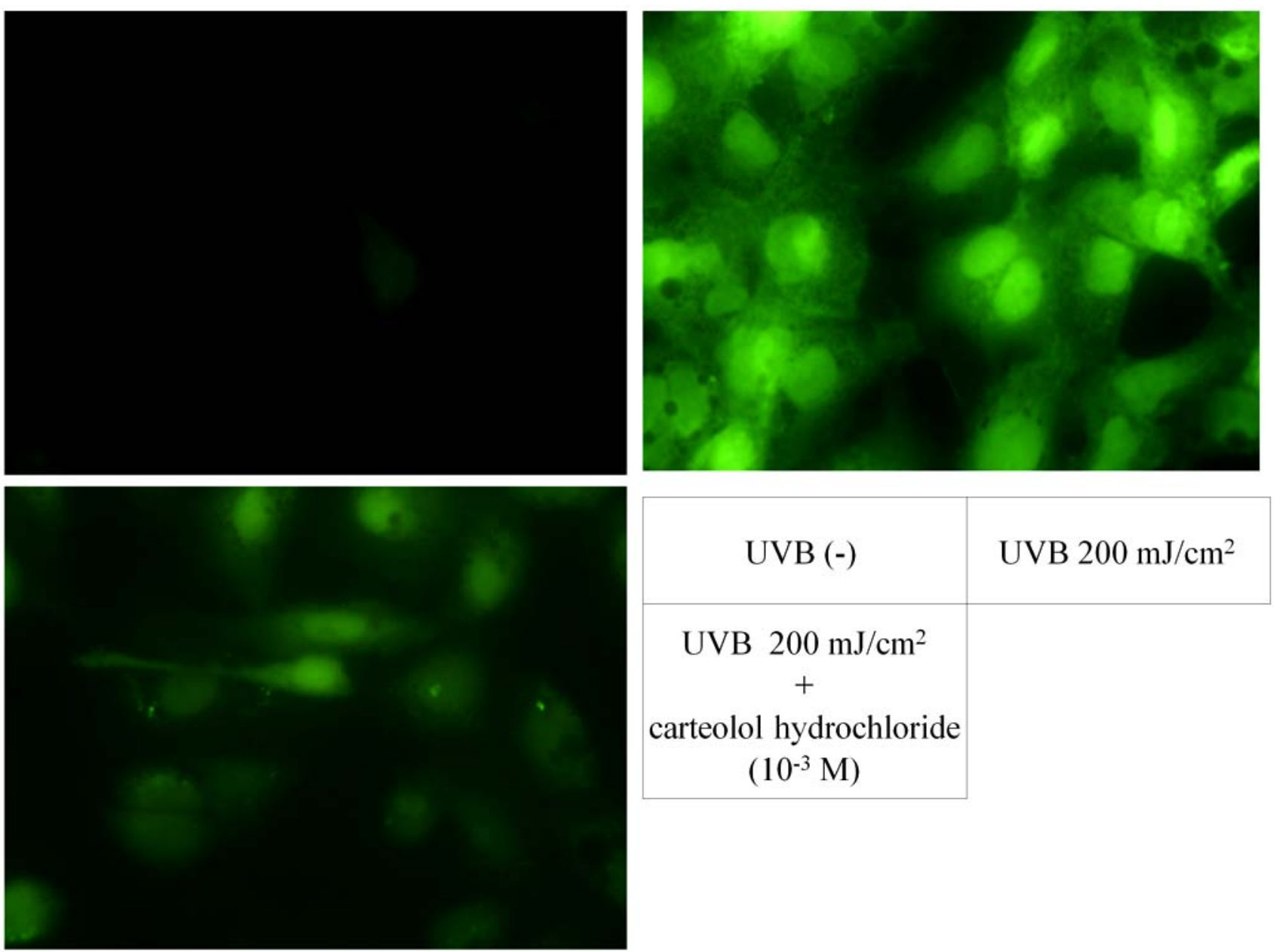

Fig. (1). Increased reactive oxygen species after ultraviolet irradiation over lens epithelial cells. Before UBV irradiation, fluorescent level was quite low (upper left). After UVB irradiation, intracellular reactive oxygen species reacted with no fluorescent 2',7'dichlorodihydrofluorescein to form fluorescent $2^{\prime}, 7^{\prime}$-dichlorofluorescein (upper right). The intensity of fluorescence decreased with pretreatment of carteolol hydrochloride before UVB exposure (lower left).

protective effect with regard to the development of cataracts because the production of ROS after UV irradiation is one of the causes of cataract formation.

All types of eye drop medication might have an effect on the eye lens in vivo. The potential effect of anti-glaucoma eye drops on lens epithelial cells is of particular importance because these products are used for periods extending over years in patients with glaucoma. Among various antiglaucoma drugs, prostaglandin analogues are reported to have effects on cataract formation. Andley et al., for example, have reported that prostaglandin $E_{2}$ suppresses and prostaglandin $\mathrm{F}_{2}$ accelerates $\mathrm{UV}$-induced cataract formation [16]. On the basis of the results of this study, these authors promoted awareness that the topical application of $\mathrm{PGF}_{2}$ analogues may induce cataracts. In addition, the authors reported that increased production of prostaglandins is involved in the development of UV-induced cataracts. Indeed, UV irradiation of cultured LECs increased the production of prostaglandins by activating cyclooxygenase $[16,17]$. For these reasons, we should be aware of the potential risks of the long-term use of anti-glaucoma eye drops containing prostaglandin analogues and $\beta$-adrenergic blockers.

Oxidative stresses are closely related to the development of cataracts. Loss of reduced glutathione [18] and increases in oxidized residues in proteins [19] and lipid peroxidation $[20,21]$ are observed in human cataracts. Among various ROS, $\mathrm{H}_{2} \mathrm{O}_{2}$ is the major oxidant contributing to cataract formation [21]. Elevated $\mathrm{H}_{2} \mathrm{O}_{2}$ concentrations are observed in both the lens and aqueous humor of the eyes of patients with cataracts $[21,22]$. In addition, lenses cultured in medium containing elevated $\mathrm{H}_{2} \mathrm{O}_{2}$ develop cataracts. Furthermore, $\mathrm{H}_{2} \mathrm{O}_{2}$ can elicit protein modifications, such as glycation, oxidation, and cross-linking, that are observed in human lenses with cataracts [23]. Among the various methods for evaluation of RPS in cells, we have used a fluorescent probe $2^{\prime}, 7^{\prime}$-dichlorodihydrofluorescein diacetate [24] for three reasons. First, the compound is commercially available and widely used to evaluate the intracellular concentration of ROS. Second, this compound reflects the intracellular concentration of various ROS including $\mathrm{H}_{2} \mathrm{O}_{2}$, $\mathrm{NO}, \mathrm{OH}$, and $\mathrm{ONOO}^{-}$. Third, not only the concentration of ROS, but also intracellular localization of ROS can be 

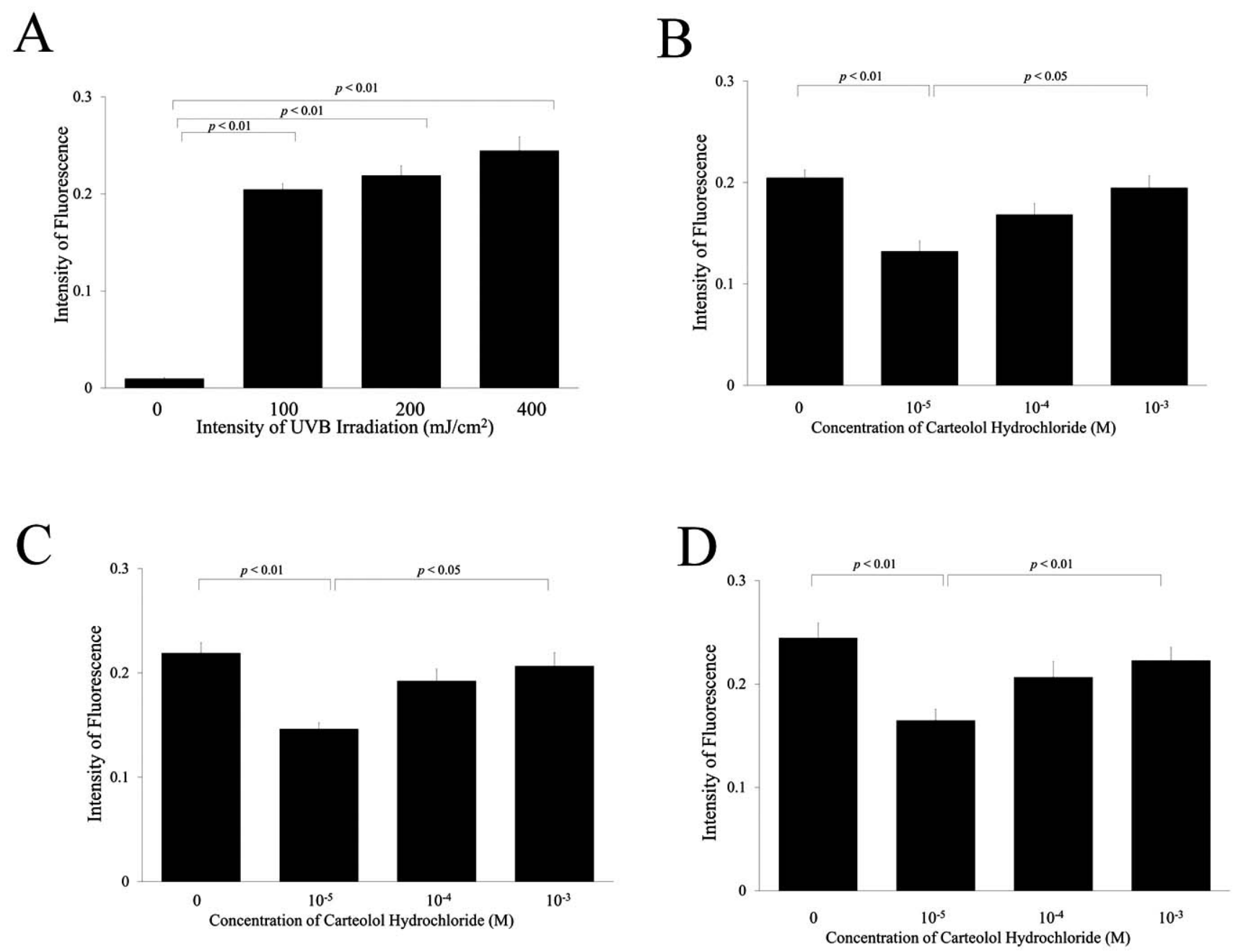

Fig. (2). Reactive oxygen species in lens epithelial cells after ultraviolet exposure. The intensity of fluorescence caused by intracellular $2^{\prime}, 7^{\prime}-$ dichlorofluorescein increased with UV exposure in a dose-dependent manner (A). Pre-treatment of carteolol hydrochloride at $10^{-3} \mathrm{M}$ significantly inhibited the intracellular fluorescence after UV exposure to 100,200 , and $400 \mathrm{~mJ} / \mathrm{cm}^{2}$, respectively (B, C, and D).

visualized under a fluorescent microscope. In the present study, a significant increase in $2^{\prime}, 7^{\prime}$-dichlorofluorescein fluorescence and a decrease in cell viability were observed after UV exposure. These changes were inhibited by the addition of carteolol hydrochloride in a dose-dependent manner. The results indicate that carteolol hydrochloride would inhibit cataract formation in an in vitro model.

Although apparently promising, the results of the present study do not necessarily suggest that topical application of carteolol hydrochloride inhibits cataract formation, due to the following reasons. First, we analyzed ROS production and cell death after UV irradiation in cultured LECs in vitro, which is not identical to cataract formation in vivo. However, we do believe that the results of the present study provide indirect evidence indicating that carteolol hydrochloride would have protective effects against cataract formation because UV irradiation and ROS production are important factors in cataract development $[25,26]$.

Second, although carteolol hydrochloride at a concentration of $10^{-3} \mathrm{M}$ inhibited ROS generation and protected against cell death after UV exposure, this concentration is considerably higher than that resulting from the topical application of carteolol hydrochloride. Fujio et al. have reported that the maximum concentration of carteolol hydrochloride in the aqueous humor after topical application is $806 \pm 147 \mathrm{ng} / \mathrm{mL}(2.46 \pm 0.45 \mathrm{mM})$ [27]. However, the concentration of carteolol hydrochloride in the lens should be much smaller than that of aqueous humor although the correct data are not reported. Accordingly, topically applied carteolol hydrochloride could not reach a concentration of $10^{-3} \mathrm{M}$ in the lens. Nevertheless, carteolol hydrochloride exhibited a dose-dependent antioxidant effect, although the effect was not statistically significant at concentrations of $10^{-4}$ and $10^{-5} \mathrm{M}$.

Third, the dosage of UBV irradiation was 100-400 $\mathrm{mJ} / \mathrm{cm}^{2}$, which was none physiological. However, the intensity of UVB irradiation at $100-400 \mathrm{~mJ} / \mathrm{cm}^{2}$ can be used in evaluation of both the production of reactive oxygen species and the cell death in in vitro studies. For example, Andley et al. evaluated the DNA repair and the cell survival of cultured lens epithelial cells after $400 \mathrm{~mJ} / \mathrm{cm}^{2}$ of UBV irradiation [28]. Yao et al. have analyzed the reactive oxygen 

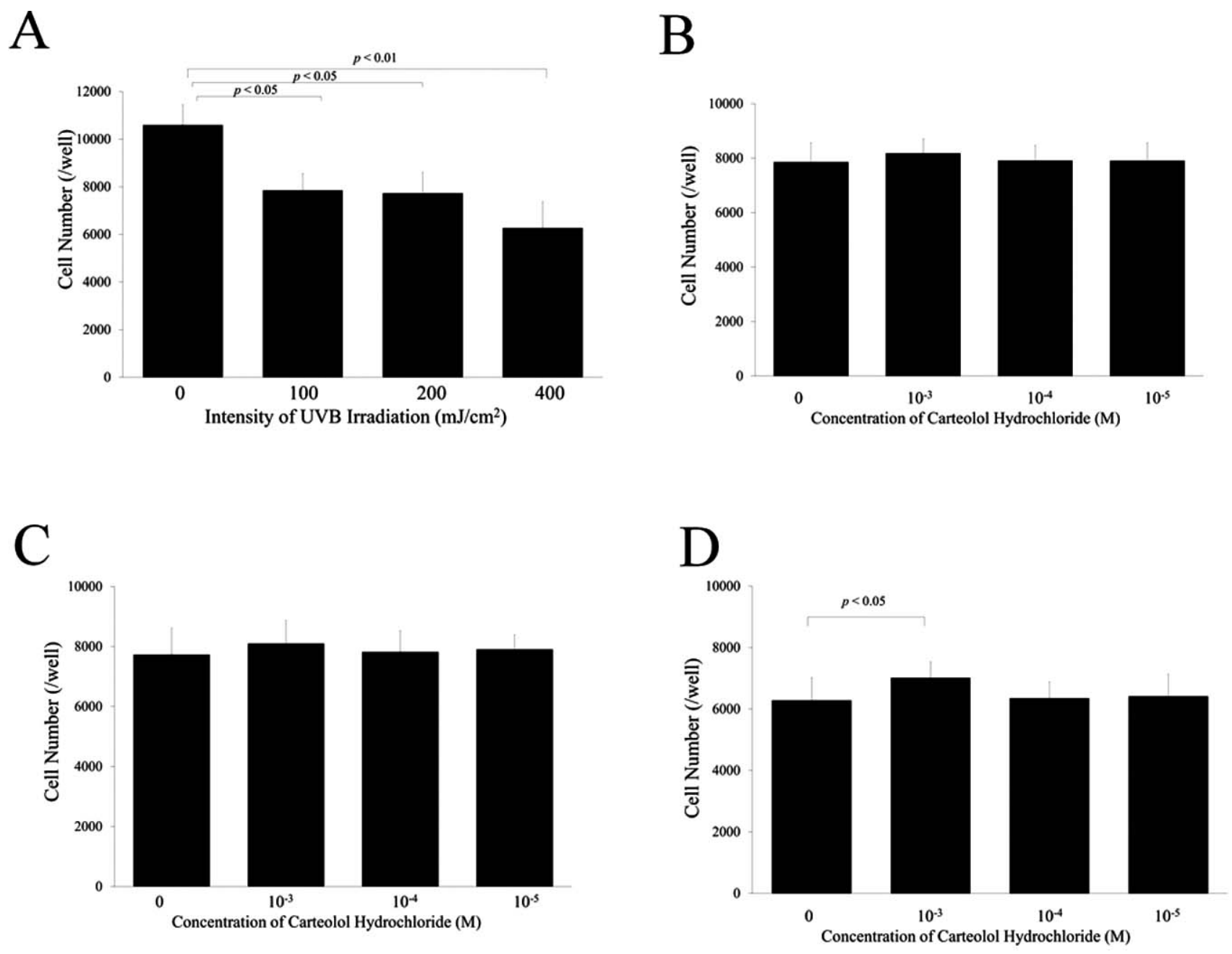

Fig. (3). Number of lens epithelial cells after exposure to ultraviolet light. The cell number decreased after UV exposure in a dose-dependent manner (A). After UV exposure of 100 and $200 \mathrm{~mJ} / \mathrm{cm}^{2}$, pre-treatment of carteolol hydrochloride had no significant effect on the cell number (B and C). In contrast, pre-treatment of carteolol hydrochloride at $10^{-3} \mathrm{M}$ significantly inhibited the intracellular fluorescence after UV exposure to $400 \mathrm{~mJ} / \mathrm{cm}^{2}$ (D).

species after UBV irradiation at $0-90 \mathrm{~mJ} / \mathrm{cm}^{2}$ [29]. Bantseev et al. have also analyzed the reactive oxygen species and cell viability after UBV irradiation ad 223 and $445 \mathrm{~mJ} / \mathrm{cm}^{2}[30]$. In this way, our experimental model of using UBV at 100 $400 \mathrm{~mJ} / \mathrm{cm}^{2}$ would be appropriate in the evaluation of both the cell death and the production of reactive oxygen species. For these reasons, we believe that the long-term application of carteolol hydrochloride may have a potential to inhibit cataract formation in vivo.

The effect of carteolol hydrochloride as a scavenger of reactive oxygen species is thought to be attributed to its molecular structure. Naito et al. have reported that various synthetic molecules containing 3,4-double bond of quinolone serve as a hydroxy radical scavenger [7]. In fact, rebamipide (2-(4-chlorobenzolamino)-3-[2(1H)-quinolon-4-yl]proppionic acid) exert its anti-ulcer action by scavenging the hydroxyl radicals in gastric mucosa. In addition, Tanito et al. by analyzing the electron spin resonance spin trapping technique have reported that carteolol hydrochloride, which possesses 3,4-double bond of quinolone in its structure, also serves as a hydroxyl radical scavenger [6].

Although we have seen an inhibitory effect of carteolol hydrochloride on the production of reactive oxygen species after 100,200 , and $400 \mathrm{~mJ} / \mathrm{cm}^{2}$, viability of the lens epithelial cells was not increased at $100 \mathrm{~mJ} / \mathrm{cm}^{2}$ of UV exposure. This result indicates that cell death after UV exposure is regulated by various factors including intracellular reactive oxygen species and direct damage on DNA and proteins. For this reason, further studies are needed to apply the results of our present study into human. The results of the present study indicated both the increase in the reactive oxygen species and the cell death after UVB irradiation was inhibited by carteolol hydrochloride depends on the conditions. Further investigation should be needed to cleat the protective effect of carteolol hydrochloride after UBV irradiation using in vivo model [31].

Cataract and glaucoma are major cause of blindness worldwide. This means that cataract surgery is not available in various parts of the world. For this reason, application of 
eye drops that may inhibit cataract formation would be beneficial for the world health. Although the protective effect of carteolol hydrochloride against UVB induced cataract has not been shown in vivo model, the results of the present study would affect the selection of anti-glaucoma drugs.

\section{ACKNOWLEDGEMENT}

This work is supported by a Ministry of Education, Science, Sports and Culture Grant for Scientific Research, 21592216 (2009-2011).

\section{ABBREVIATIONS}

LEC $=$ Lens epithelial cell
UVB $=$ Ultraviolet $\mathrm{B}$
ROS $=$ Reactive oxygen species
PBS $=$ Phosphate-buffered saline

\section{REFERENCES}

[1] Shiose Y, Kitazawa Y, Tsukahara S, et al. Epidemiology of glaucoma in Japan--a nationwide glaucoma survey. Jpn J Ophthalmol 1991; 35: 133-55.

[2] Suzuki Y, Iwase A, Araie M, et al. Risk factors for open-angle glaucoma in a Japanese population: the Tajimi Study. Ophthalmology 2006; 113: 1613-7.

[3] Yu ZK, Chen YN, Aihara M, Mao W, Uchida S, Araie M. Effects of $\beta$-adrenergic receptor antagonists on oxidative stress in purified rat retinal ganglion cells. Mol Vis 2007; 13: 833-9.

[4] Melena J, Osborne NN. Metipranolol attenuates lipid peroxidation in rat brain: a comparative study with other antiglaucoma drugs. Graefes Arch Clin Exp Ophthalmol 2003; 241: 827-33.

[5] Asayama K, Dobashi K, Hayashibe H, Kato K. Effects of $\beta$ adrenergic blockers with different ancillary properties on lipid peroxidation in hyperthyroid rat cardiac muscle. Endocrinol Jpn 1989; 36: 687-94

[6] Tanito M, Takanashi T, Kaidzu S, Yoshida Y, Ohira A. Cytoprotective effects of rebamipide and carteolol hydrochloride against ultraviolet B-induced corneal damage in mice. Invest Ophthalmol Vis Sci 2003; 44: 2980-5.

[7] Naito Y, Yoshikawa T, Tanigawa $\mathrm{T}$, et al. Hydroxyl radical scavenging by rebamipide and related compounds: electron paramagnetic resonance study. Free Radic Biol Med 1995; 18: 11723.

[8] Ono H, O'Hara N. A study on the cardiodepressant action of a $\beta$ blocking agent carteolol in heart-lung preparation of the dog. Jpn Circ J 1984; 48: 1030-44.

[9] Chrisp P, Sorkin EM. Ocular carteolol. A review of its pharmacological properties, and therapeutic use in glaucoma and ocular hypertension. Drugs Aging 1992; 2: 58-77.

[10] Kuwahara K, Oizumi N, Fujisawa S, Tanito M, Ohira A. Carteolol hydrochloride protects human corneal epithelial cells from UVBinduced damage in vitro. Cornea 2005; 24: 213-20.

[11] Soderberg PG. Experimental cataract induced by ultraviolet radiation. Acta Ophthalmol Suppl 1990; 1-75.
[12] Sliney DH. Epidemiological studies of sunlight and cataract: the critical factor of ultraviolet exposure geometry. Ophthalmic Epidemiol 1994; 1: 107-19.

[13] Dolin PJ. Ultraviolet radiation and cataract: a review of the epidemiological evidence. Br J Ophthalmol 1994; 78: 478-82.

[14] Ayala M, Strid H, Jacobsson U, Soderberg PG. p53 expression and apoptosis in the lens after ultraviolet radiation exposure. Invest Ophthalmol Vis Sci 2007; 48: 4187-91.

[15] Linetsky M, Ortwerth BJ. Quantitation of the reactive oxygen species generated by the UVA irradiation of ascorbic acid-glycated lens proteins. Photochem Photobiol 1996; 63: 649-55.

[16] Andley UP, Fritz C, Morrison AR, Becker B. The role of prostaglandins E2 and F2 alpha in ultraviolet radiation-induced cortical cataracts in vivo. Invest Ophthalmol Vis Sci 1996; 37: $1539-48$.

[17] Andley UP, Becker B, Hebert JS, Reddan JR, Morrison AR, Pentland AP. Enhanced prostaglandin synthesis after ultraviolet-B exposure modulates DNA synthesis of lens epithelial cells and lowers intraocular pressure in vivo. Invest Ophthalmol Vis Sci 1996; 37: 142-53.

[18] Giblin FJ. Glutathione: a vital lens antioxidant. J Ocul Pharmacol Ther 2000; 16: 121-35.

[19] Hains PG, Truscott RJ. Post-translational modifications in the nuclear region of young, aged, and cataract human lenses. J Proteome Res 2007; 6: 3935-43.

[20] Babizhayev MA, Costa EB. Lipid peroxide and reactive oxygen species generating systems of the crystalline lens. Biochim Biophys Acta 1994; 1225: 326-37.

[21] Bhuyan KC, Bhuyan DK, Podos SM. Lipid peroxidation in cataract of the human. Life Sci 1986; 38: 1463-71.

[22] Spector A, Garner WH. Hydrogen peroxide and human cataract. Exp Eye Res 1981; 33: 673-81.

[23] Zigler JS, Jr., Huang QL, Du XY. Oxidative modification of lens crystallins by $\mathrm{H}_{2} \mathrm{O}_{2}$ and chelated iron. Free Radic Biol Med 1989; 7: 499-505.

[24] Krejsa CM, Schieven GL. Detection of oxidative stress in lymphocytes using dichlorodihydrofluorescein diacetate. Methods Mol Biol 2000; 99: 35-47.

[25] Balasubramanian D. Ultraviolet radiation and cataract. J Ocul Pharmacol Ther 2000; 16: 285-97.

[26] Truscott RJ. Age-related nuclear cataract-oxidation is the key. Exp Eye Res 2005; 80: 709-25.

[27] Fujio N, Kusumoto N, Odomi M. Ocular distribution of carteolol after single and repeated ocular instillation in pigmented rabbits. Acta Ophthalmol (Copenh) 1994; 72: 688-93.

[28] Andley UP, Song Z, Mitchell DL. DNA repair and survival in human lens epithelial cells with extended lifespan. Curr Eye Res 1999; 18: 224-30.

[29] Yao J, Liu Y, Wang X, et al. UVB radiation induces human lens epithelial cell migration via NADPH oxidase-mediated generation of reactive oxygen species and up-regulation of matrix metalloproteinases. Int J Mol Med 2009; 24: 153-9.

[30] Bantseev V, Youn HY. Mitochondrial "movement" and lens optics following oxidative stress from UV-B irradiation: cultured bovine lenses and human retinal pigment epithelial cells (ARPE-19) as examples. Ann N Y Acad Sci 2006; 1091: 17-33.

[31] Mody VC, Kakar M, Elfving A, Lofgren S. Drinking water supplementation with ascorbate is not protective against UVR-Binduced cataract in the guinea pig. Acta Ophthalmol 2008; 86: 18895 .

This is an open access article licensed under the terms of the Creative Commons Attribution Non-Commercial License (http: //creativecommons.org/licenses/by$\mathrm{nc} / 3.0 /$ ) which permits unrestricted, non-commercial use, distribution and reproduction in any medium, provided the work is properly cited. 